\title{
Low-temperature magnetoresistance in polycrystalline manganites: connectivity versus grain size
}

\author{
A. de Andrés, ${ }^{\text {a) }}$ M. García-Hernández, J. L. Martínez, and C. Prieto \\ Instituto de Ciencia de Materiales de Madrid, Consejo Superior de Investigaciones Científicas, \\ Campus de Cantoblanco, E-28049 Madrid, Spain
}

(Received 3 February 1999; accepted for publication 22 April 1999)

\begin{abstract}
We have performed magnetic and transport measurements on $\mathrm{La}_{0.7} \mathrm{Ca}_{0.3} \mathrm{MnO}_{3}$ polycrystalline pellets and thin films. We propose a model that reproduces very well the low-field and high-field magnetoresistance at low temperature. The decrease of the resistance at high fields is modeled by the opening of new conduction channels in parallel due to the ordering of Mn spins blocked at the grain surface. We found that high-field magnetoresistance is independent of grain size, at least down to $12 \mathrm{~nm}$, but depends strongly on the connectivity between grains. (C) 1999 American Institute of Physics. [S0003-6951(99)04524-6]
\end{abstract}

Manganese perovskites $\mathrm{La}_{1-x} \mathrm{~A}_{x} \mathrm{MnO}_{3} \quad(\mathrm{~A}=\mathrm{Ca}, \mathrm{Sr}$, $\mathrm{Ba} . .$. ) have recently attracted much interest because of their potential magnetic applications using their high magnetoresistance effect. ${ }^{1}$ Ca-doped compounds with $0.15<x<0.5$ present an insulator-to-metal transition, at $T_{\mathrm{IM}}$, and para to ferromagnetic (FM) ordering, at $T_{c}$, both about $265 \mathrm{~K}$ for $x=0.3$. Single-crystal and epitaxial films magnetoresistance (MR) is maximum near $T_{\mathrm{IM}}\left(\approx T_{c}\right)$ and almost zero at low temperatures. On the contrary, polycrystalline compounds present high MR down to low temperatures. ${ }^{2,3}$ The effect of grain boundaries and grain size have been extensively studied. Several authors report on the decrease of the $T_{\mathrm{IM}}$ for granular systems ${ }^{4}$ or films with microcracks ${ }^{5}$ and related that to grain size effects ${ }^{3}$ or to the existence of insulating regions at grain boundaries. ${ }^{4} \mathrm{Kim}$ et al. suggest that intergranular resistance is probably the origin of the discrepancies between infrared and direct current $(\mathrm{dc})$ resistivities. ${ }^{6}$ Low-field MR at low temperature is now established to be a spin-dependent scattering process at grain boundaries. ${ }^{2,7-9}$ The description of the polycrystalline manganites MR in terms of intrinsic and extrinsic components attempts to explain the observed increase in the high-field MR (HFMR), ${ }^{10,11}$ when the magnetization is almost saturated. However, a model that quantitatively reproduces the measured resistivity and MR as a function of the interfaces and grain size is still lacking.

In order to assess the effects of connectivity between grains on the magnetic and transport properties, we have prepared a set of ceramic samples that, having the same grain size, only differ in the degree of conglomeration. $\mathrm{La}_{0.7} \mathrm{Ca}_{0.3} \mathrm{MnO}_{3}$ powder was obtained by a standard ceramic method. The powder, with mean grain size of $1-3 \mu \mathrm{m}$ as seen by optical microscopy, was pressed into pellets and then sintered at $200,500,800$, and $1100{ }^{\circ} \mathrm{C}$ for $4 \mathrm{~h}$. The effect of sintering is to "glue" one grain to another, varying the connectivity between them. Grain size effects are checked by comparing these pellets to polycrystalline thin films grown by dc sputtering at room temperature on $\mathrm{Si}(100)$ substrates in a mixed $\mathrm{Ar}$ and $\mathrm{O}_{2}$ atmosphere (ratio 4:1) and then annealed at $850{ }^{\circ} \mathrm{C}$ for $600 \mathrm{s.}{ }^{12}$ The films' atomic force microscopy (AFM) images show grains of rather spherical shape with mean diameters between 12 and $80 \mathrm{~nm}$ (inset of Fig. 1).

a)Electronic mail: ada@icmm.csic.es
X-ray diffractograms were obtained for all pellets showing identical lattice parameters and peak widths (Fig. 1). Grazing incidence and standard x-ray diffraction of the thin films reveal their polycrystalline character and expected lattice parameters for Ca concentration of about $28 \%$.

The magnetization measurements were performed in a SQUID magnetometer. The electric transport measurements were performed in a PPMS (Quantum Design) system always on fresh samples.

The upper part of Fig. 2(a) shows the magnetic transition temperature of the different pellets, which is identical for all of them. The lower part presents the measured resistance of the same samples at 0 and $9 \mathrm{~T}$, normalized by their value at $300 \mathrm{~K}$ and $0 \mathrm{~T}$. Figure 2(b) shows the same information for a thin film with mean grain diameter of $20 \mathrm{~nm}$, which presents a slightly lower $T_{c}$ related to the lower Ca content. In both cases, magnetization at $5 \mathrm{~K}$ is practically saturated at about $1 \mathrm{~T}$. As the sintering temperature of the pellets decreases $T_{\mathrm{IM}}$ decreases, the resistance at $300 \mathrm{~K}$ increases several orders of magnitude (not seen in the figure because normalized at their $300 \mathrm{~K}$ values) and $R(T) / R(300 \mathrm{~K})$ dramatically increases at low temperatures. The decrease of $T_{\mathrm{IM}}$ and increase of the resistance, for samples with the same $T_{c}$, have been previously observed ${ }^{4,11}$ and either have been overlooked or related to grain size effects. Notice that all pellets in Fig 2(a) have been obtained from the same powder

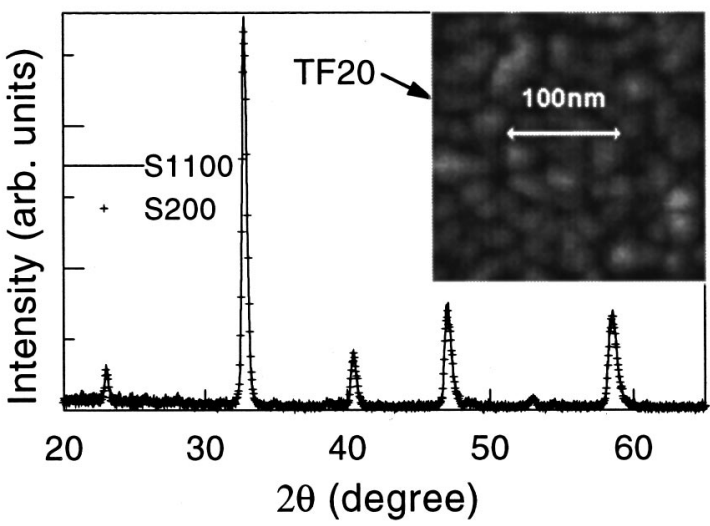

FIG. 1. XRD patterns of pellets sintered at 1100 and $200^{\circ} \mathrm{C}$. Inset: AFM image of a thin film (TF20) with mean grain diameter $=20 \mathrm{~nm}$. 


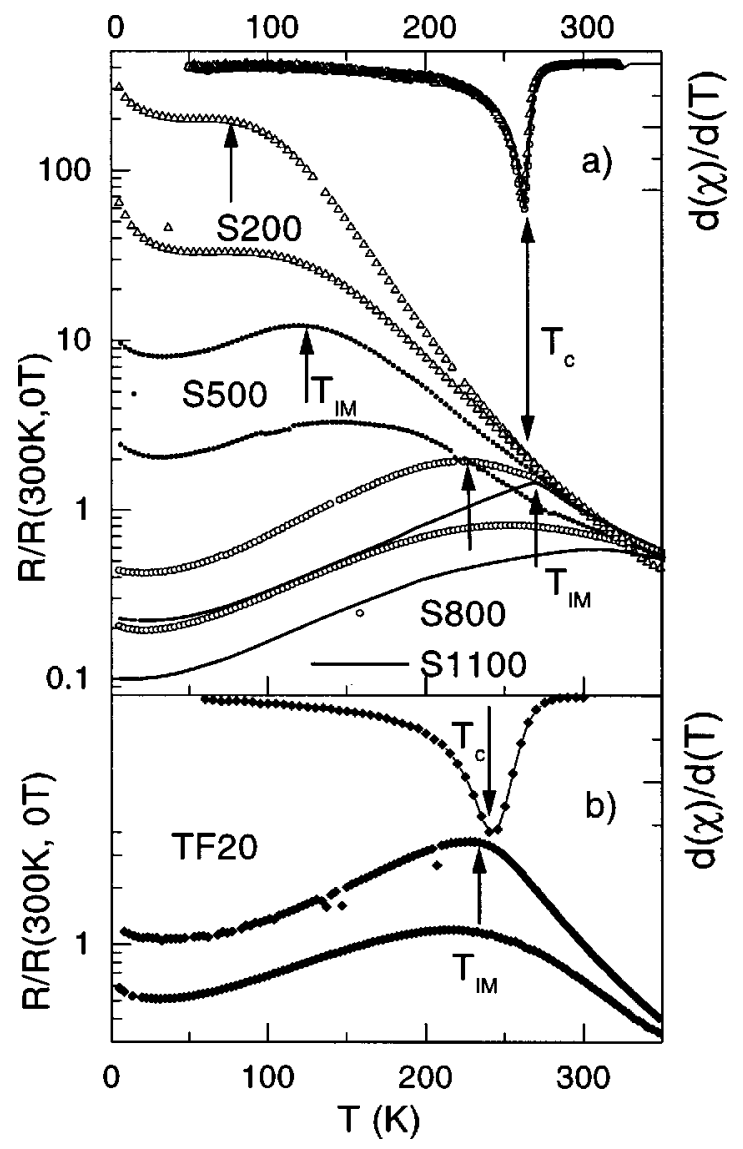

FIG. 2. (a) Derivative of the susceptibility of representative pellets (sintered at different temperatures: $200,500,800$, and $1100^{\circ} \mathrm{C}$ ) and their normalized resistance at 0 and $9 \mathrm{~T}$. (b) Derivative of the susceptibility and normalized resistance at 0 and $9 \mathrm{~T}$ of a thin film with $20 \mathrm{~nm}$ grains.

with grain size about $1-3 \mu \mathrm{m}$. The sintering does not change the grain size but it increases the connectivity between grains, that is, the fraction of the grain surface which is in a sufficient contact to show a metallic conductivity below $T_{c}$.

In order to explain the observed behavior of the electrical conduction in polycrystalline samples, we propose the existence of two kinds of conduction channels parallel connected. One kind is related to the transport properties of the system and is achieved through the contact areas between grains. The other shows energy barriers at all temperatures due to poor connectivity, disorder, and contamination at the grain surface that inhibit metallic conduction. The details of this model, that reproduces the measured $R(T)$ for all samples, together with the parameters of the fits will be reported elsewhere. We observe that, the higher the annealing temperature for the pellet, the larger is the effective section for channels with metallic conductivity at low temperature.

Note that S1100 pellet (grains about $2000 \mathrm{~nm}$ ) and TF20 thin film (grains about $20 \mathrm{~nm}$ ) samples present $T_{\text {IM }}$ similar to $T_{c}$, as expected from the double exchange mechanism, indicating the good quality of the samples and a good connectivity. In fact MR at $5 \mathrm{~K}$ of S1100 and TF20 are identical (Fig. 3). The slope of the HFMR at $5 \mathrm{~K}$ reported for different manganites with grain sizes above $500 \mathrm{~nm}$ are all nearly identical (around 0.23$)^{11}$ and coincides with our data for S1100 sample and for all our thin films (grain diameters between 80 and $12 \mathrm{~nm}$ ). On the contrary, pellets with lower $T_{\mathrm{IM}}$ temperatures (lower connectivity) present a higher slope Downloaded 09 Mar 2010 to 161.111.180.191. Redistribution subje

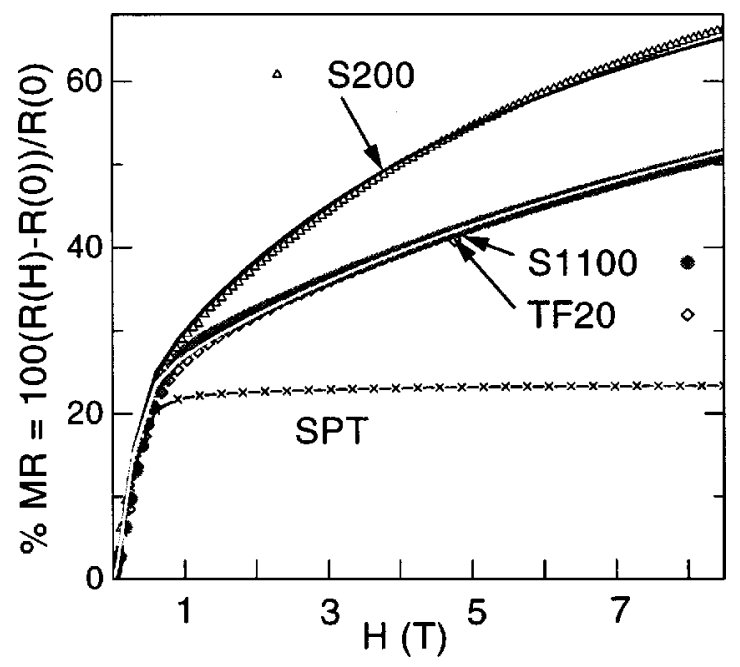

FIG. 3. Magnetoresistance at $5 \mathrm{~K}$ of two pellets and one thin film. Crosses: MR obtained from the measured magnetization of the pellets. Continuous lines (black and white) are the fits to the measured MR of the three samples.

of the HFMR (e.g., "S200" in Fig. 3). In summary, MR above $1 \mathrm{~T}$ (when the magnetization is almost saturated) does not depend on the grain size (at least down to $12 \mathrm{~nm}$ ) and the HFMR slope is higher when the connectivity is poor.

Figure 3 shows the measured and calculated MR for the pellets annealed at $1100{ }^{\circ} \mathrm{C}(\mathrm{S} 1100)$ and $200{ }^{\circ} \mathrm{C}$ (S200) and the thin film TF20. The model considers the following mechanism: At low temperature, the metallic channels give rise to a MR which is determined by the spin-dependent tunneling between grains that depends on the angle formed by the magnetic moments of these grains. ${ }^{11}$ The variation of this angle with the external magnetic field is given by the measured magnetization $M(H)$. Therefore, the conductivity for metallic channels can be described by the following expression: $\sigma_{G}(H)=\sigma_{G}(0)+C\left(\left(M(H) / M_{s}\right)^{2}\right.$, where $M_{s}$ is the saturated magnetization, $C$ is a constant for a given temperature, and $R_{G}(H) / R_{G}(0)=1 /(1+C[(M(H) /$ $\left.\left.M_{s}\right)^{2} / \sigma_{G}(0)\right]$. We define $\operatorname{MR}(H)=100\left[R_{G}(H) / R_{G}(0)-1\right]$ which is represented by crosses in Fig. 3. At the grain surface, Mn spins can be blocked, for example, because their environment is unbalanced or because of magnetic anisotropy. In fact, recent calculations demonstrate that Mn ions of the outmost layer have their $e_{g}$ orbitals split and their charge state modified. ${ }^{13}$ Magnetic measurements are not sensitive to these disordered $\mathrm{Mn}$ spins because the fraction is very small, but the conduction at low temperature is drastically reduced because one distorted $\mathrm{Mn}$ closes that conduction channel. The main hypothesis of our model is that the magnetic field $(H)$ aligns Mn spins blocked at the surface in a linear way, opening new conduction channels. Therefore, the effective section for electric conduction is proportional to the applied magnetic field: $S_{\text {new }}(H=f . H$ ( $f$ is a constant). As both kind of channels are parallel connected, the inverse of the resistances are added. Those channels that were already metallic at $H=0$ have an effective section $S_{G}$, while those that have become metallic by the application of a magnetic field present an effective section $S_{\text {new }}$. The model finally leads to the following expression: $R(H) / R(0)=1 /\left\{1+\left[C / \sigma_{G}(0)\right]\right.$ $\left.\times\left[M(H) / M_{s}\right]^{2}\right\}\left(1+f . H / S_{G}\right)$, where $S_{G}$ depends on the connectivity and contamination of the sample. Fits in Fig. 3 to AIP license or copyright; see http://apl.aip.org/apl/copyright.jsp 
render values: $C / \sigma_{G}(0)=0.33$ (for the three samples) and $f / S_{G}=0.068$ (for samples S1100 and TF20) and 0.14 (for sample S200) which is consistent with the idea that $S_{G}(\mathrm{~S} 1100)>S_{G}(\mathrm{~S} 200)$. Note that HFMR is not linear and that our model fits perfectly the data in the whole measured magnetic field range for all samples with only one parameter $\left(f / S_{G}\right)$. Within this scenario, the steady decrease of the resistance at high fields is explained by the increase of the effective section for metallic conduction by the alignment of pinned Mn magnetic moments at the surface of the grains. The slope of the HFMR depends on the ratio between the metallic to nonmetallic sections that, in turn, depends on the connectivity between grains and on the stiffness of the blocked Mn spins at the surface. The present experimental data (comparing pellets and thin films) as well as our model do not show any dependence with grain size in the present range.

In summary, in the present letter, we have shown that the interpretation of transport data has to be very carefully done. It has to be taken into account that the conduction is achieved by parallel channels. The section for metallic paths is determined by the connectivity between grains that depends on the degree of sintering and/or the contamination of the surface of the grains. The effect of an external magnetic field is to align blocked spins at the surface of the grains in a linear way opening new conduction channels that were broken, decreasing considerably the resistance (in cases more than $50 \%$ between 1 and $9 \mathrm{~T}$ ), while the magnetization remains nearly constant. This effect is not related to the grain size or to the width of the claimed disordered skin of the grains but to the differences in the effective section for metallic conduction of the samples and on the stiffness of the blocked Mn spins at the grain surface.

The authors thank L. Brey and F. Guinea for fruitful discussions and L. Vazquez for AFM measurements. This work was supported by CICyT under Contract Nos. MAT960395-CP and MAT97-725.

${ }^{1}$ S. Jin, T. H. Tiefel, M. McCormack, R. A. Fastnacht, R. Ramesh, and L. H. Chenl, Science 264, 413 (1994).

${ }^{2}$ H. Y. Hwang, S. W. Cheong, N. P. Ong, and B. Batlogg, Phys. Rev. Lett. 77, 2041 (1996).

${ }^{3}$ A. Gupta, G. Q. Gong, G. Xiao, P. R. Duncombe, P. Lecoeur, P. Trouilloud, Y. Y. Wang, V. P. Dravid, and J. Z. Sun, Phys. Rev. B 54, 15629 (1996).

${ }^{4}$ S. Pignard, H. Vincent, J. P. Senateur, K. Frohlich, and J. I. Souc, Appl. Phys. Lett. 73, 999 (1998).

${ }^{5}$ K. M. Satyalakshmi, B. Fisher, L. Patlagan, G. Koren, E. Sheriff, R. Prozorov, and Y. Yeshurun, Appl. Phys. Lett. 73, 402 (1998).

${ }^{6}$ K. H. Kim, J. Y. Gu, H. S. Choi, D. J. Eom, J. H. Jung, and T. W. Noh, Phys. Rev. B 55, 4023 (1997).

${ }^{7}$ X. W. Li, A. Gupta, G. Xiao, and G. Q. Gong, Appl. Phys. Lett. 71, 1124 (1997).

${ }^{8}$ R. Shreekala, M. Rajeswari, K. Ghosh, A. Goyal, J. Y. Gu, C. Kwon, Z. Trajanovic, T. Boettcher, R. L. Greene, R. Ramesh, and T. Venkatesan, Appl. Phys. Lett. 71, 282 (1997).

${ }^{9}$ J. M. D. Coey, Philos. Trans. R. Soc. London, Ser. A 356, 1539 (1998).

${ }^{10}$ Ll. Balcells, J. Fontcuberta, B. Martínez, and X. Obradors, Phys. Rev. B 58, 14697 (1998).

${ }^{11}$ P. Raychaudhuri, K. Sheshaadri, P. Taneja, S. Bandyopaghyay, P. Ayyub, A. K. Nigam, and R. Pinto, Phys. Rev. B (in press).

${ }^{12}$ M. García-Hernández, J. L. Martínez, A. de Andrés, L. Vázquez, E. Herrero, and J. M. Alonso, J. Magn. Magn. Mater (to be published).

${ }^{13}$ M. J. Calderón, L. Brey, and F. Guinea (unpublished). 